\title{
La quaestio "An terra moveatur an quiescat" di Giovanni Regiomontano
}

\author{
Pietro Daniel Omodeo (Venezia) e Alberto Bardi (Gerusalemme) ${ }^{1}$
}

L'An terra moveatur an quiescat è una curiosa trattazione rinascimentale del problema del moto terrestre, attribuita all'illustre astronomo matematico tedesco Giovanni Regiomontano (Johannes Müller da Königsberg, 1436-1476) dai suoi successori. ${ }^{2}$ II matematico Giovanni Schöner (1477-1547) la pubblicò nel 1533 nella città di Norimberga, dove era attivo, quale secondo capitolo di un suo Opusculum geographicum. ${ }^{3}$ Agli occhi di Schöner la discussione dell'immobilità della terra, assieme a quella della sua sfericità e a nozioni base di geografia astronomica, era propedeutica per una trattazione specifica delle regioni del globo terrestre, con le loro denominazioni e coordinate geografiche, a cui è dedicata la seconda parte dell'Opusculum. Sin dal 1514 le ipotesi geocinetiche ed eliocentriche di Niccolò Copernico avevano cominciato a diffondersi a partire dai circoli universitari di Cracovia, ma l'opera maggiore dell'astronomo, il De revolutionibus orbium coelestium, che avrebbe rivoluzionato la teoria planetaria, era ancora incompleta. ${ }^{4}$ Sarebbe apparsa nella stessa Norimberga dieci anni dopo la stampa dell'Opusculum grazie a Giorgio Gioacchino Retico (1514-1574), giovane professore di matematica di Wittenberg, il quale era andato a conoscere Copernico in Varmia proprio su suggerimento di Schöner e aveva in seguito procurato il manoscritto del De revolutionibus allo stampatore Giovanni Petreio (ca. 1496-1550). In segno di riconoscenza per averlo così bene indirizzato, Retico aveva dedicato a Schöner la Narratio prima (Danzica, 1540), prima presentazione della teoria copernicana alla repubblica delle lettere. ${ }^{5}$ Questo è il contesto scientifico e la temperie culturale nel quale si colloca la stampa del frammento sul moto terrestre attribuito a Regiomontano. ${ }^{6}$ Siccome la pubblicazione si colloca ad un crocevia di idee

\footnotetext{
${ }^{1}$ Desideriamo ringraziare le istituzioni che hanno reso questo lavoro possibile: il contesto ERC EarlyModernCosmology finanziato dal programma di ricerca e innovazione Horizon 2020 dell'Unione Europea (GA n. 725883), la Polonsky Academy del Van Leer Institute di Gerusalemme e la Dibner Library of the History and Science and Techonogy, Washington DC.

${ }^{2}$ Discutiamo più ampiamente i contesti culturali del testo oggetto di studio in un saggio di prossima pubblicazione: Alberto Bardi e Pietro Daniel Omodeo, "The Disputational Culture of Renaissance Astronomy: Johannes Regiomontanus's An terra moveatur an quiescat," in Early Modern Disputations and Dissertations in an Interdisciplinary and European Context, ed. Robert Seidel (Leiden: Brill, in press).

${ }^{3}$ Il titolo esteso è loannis Schoneri Carolostadii Opusculum Geographicum ex diversorum libris ac cartis summa cura et diligentia collectum, accommodatum ad recenter elaboratum ab eodem globum descriptionis terrenae ([Nuremberg]: [Petreius], 1533).

${ }^{4}$ Marian Biskup, Regesta copernicana (Calendar of Copernicus' Papers) (Wrocław: Zakl. Nar. Im Ossolińskich, 1973), pp. 63-64, n. 91. Sulla prima recezione di Copernico si veda Pietro Daniel Omodeo, Copernicus in the Cultural Debates of the Renaissance. Reception, Legacy, Transformation (Boston-Leiden: Brill, 2014), pp. 11-65.

${ }^{5}$ Cf. Jarosław Włodarczyk, Introduction a Georg Joachim Rheticus, Narratio prima or First Account of the Books On the Revolutions by Nicolaus Copernicus (Warsaw: University of Warsaw Press, 2015), pp. 9-70, in particolare p. 13.

${ }^{6}$ Qui faremo riferimento all'edizione facsimile Johannes Regiomontanus, Opera collectanea (Osnabrück: Zeller, 1972), a cura di Felix Schmeidler, pp. 37-39, ma abbiamo consultato anche una copia a stampa dell'Opusculum originale del 1533, conservata alla Dibner Library of the History and Science and Techonogy, Washington DC. Cf. Bern Dibner, "The Burndy Library in Mitosis. (Contemporary Collectors, XLIX)." The Book Collector 26 (1977), p. 12; Robert Westman,
} 
astronomiche e dibattiti cosmologici di fondamentale importanza per la storia della scienza moderna, una sua riconsiderazione è auspicabile. Ci proponiamo di ricapitolarne i contenuti e pubblicare qui la prima traduzione in una lingua moderna. Indipendentemente dalla correttezza o meno dell'attribuzione a Regiomontano, l'ascrizione stessa e il contesto di ricezione sono sufficienti a farne un documento dell' accendersi di discussioni sul moto terrestre in contesto astronomico negli anni che precedono la pubblicazione del De revolutionibus copernicano. Inutile aggiungere che il rilievo delle posizioni espresse in una controversia non vanno misurate nei termini anacronistici di un'adesione alla tesi del moto terrestre o di un rigetto della stessa. Dal nostro punto di vista è la possibilità di documentare un dibattito o di aggiungere elementi di valutazione che merita l'attenzione dello storico della cultura scientifica, indipendentemente dai suoi esiti storici.

II testo in questione ha la veste prettamente scolastica di una quaestio disputata. ${ }^{7}$ Più precisamente, Regiomontano lo presenta quale quaestio de motu locali. La disputa, incentrata su un problema specifico quale, nel nostro caso, il moto terrestre, era tipico genere universitario. Infatti, la discussione degli argomenti pro e contra determinate tesi era esercizio fondamentale dell'educazione medievale e rinascimentale. ${ }^{8}$ Nonostante Regiomontano sia stato spesso interpretato quale dotto umanista del cosiddetto 'Rinascimento matematico', estraneo alle forme 'barbare' della scolastica, testi come l'An terra moveatur attestano piuttosto la continuità delle varie espressioni della cultura scientifica quattrocentesca. ${ }^{9}$ L'attenzione del Regiomontano per le forme e i contenuti dell'insegnamento universitario è altresì attestata da una sua famosa orazione sulla dignità degli studi matematici, da lui pronunciata a Padova nel 1464 in occasione di un corso di astronomia sferica basato su Alfragano. Petreio l'avrebbe pubblicata nel 1537 col titolo di Oratio introductoria in omnes scientias mathematicas, ponendola a premessa di due classici dell'astronomia islamica, i Rudimenta astronomica di Alfragano nella traduzione di Giovanni da Siviglia e il De motu stellarum di Albategno nella versione di Platone da Tivoli. ${ }^{10}$ Schöner, responsabile della revisione ed edizione di tali opere, utilizzò come base testuale un codice di Regiomontano. ${ }^{11}$

Non è mancato chi mettesse in dubbio addirittura l'attribuzione della disputa a Regiomontano. L'autore della più significativa prosopografia dello scienziato, Ernst Zinner, la considerò spuria ritenendo che Schöner fosse caduto in errore oppure avesse voluto ammantare

Copernicus and the Astrologers: Dibner Library Lecture, December 12, 2013 (Washington DC: Smithsonian Libraries, 2016), pp. 44-48.

${ }^{7}$ Brian Lawn, The Rise and Decline of the Scholastic 'Quaestio disputata' with Special Emphasis on Its Use in the Teaching of Medicine and Science (Leiden: Brill, 1993).

${ }^{8}$ Anche a Vienna, l'università a cui Regiomontano fu maggiormente legato. Si veda Michael H. Shank, "Scientific tradition in Fifteenth-Century Vienna" in Tradition, Transmission, Transformation: Proceedings of Two Conferences on PreModern Science Held at the University of Oklahoma, a cura di F. Jamil Ragep and Sally P. Ragep (Leiden: Brill, 1996), pp. 117-120.

9 L'immagine di Regiomontano umanista matematico è stata magnificata da Paul Lawrence Rose, The Italian Renaissance of Mathematics: Studies on Humanists and Mathematicians from Petrarch to Galileo (Genève: Droz 1975), pp. 90-117.

${ }^{10}$ Una discussione dell'orazione e della sua recezione potrà essere trovata nel saggio di prossima pubblicazione Pietro Daniel Omodeo, "Johannes Regiomontanus and Erasmus Reinhold on the History of Astronomy: Two Concepts of Renaissance", in Translations and Their Consequences, 8th-16th Centuries, ed. by Sonja Brentjes (Berlin: Edition Open Access, in stampa).

${ }^{11}$ Codice Nurembergensis Cent. VI 21. Cf. Ernst Zinner, Regiomontanus: His Life and Work (Amsterdam: NorthHolland, 1990), p. 216. 
l'argomento del prestigio della maggiore autorità scientifica della generazione precedente. ${ }^{12}$ Tali dubbi o sospetti paiono però eccessivi sotto vari punti di vista. Innanzitutto la confutazione del moto terrestre non era controversa negli anni Trenta del Cinquecento e dunque non richiedeva il patrocinio di una auctoritas moderna potendo già contare su quelle classiche, in primis Tolomeo e Aristotele. Inoltre Schöner godeva di una posizione privilegiata da cui asseverare l'opera e le idee di Regiomontano in quanto apparteneva ad una comunità scientifica che aveva beneficiato dell'insegnamento dei suoi allievi o collaboratori diretti, tra cui Bernard Walther (1430-1504). In considerazione del contesto culturale di Norimberga e della familiarità degli scienziati tedeschi con l'opera di Regiomontano, ci pare altamente implausibile che un'indebita attribuzione potesse passare per buona senza rimostranze. La nostra valutazione non vuole certo sostituire la filologia d'autore ma integrarla in prospettiva storico-culturale. Non essendo stato trovato un testo equivalente negli autografi del nostro, resta un margine di dubbio se il testo stampato da Schöner sia una copia di una stesura ad opera della mano del nostro oppure il frutto di appunti presi da un socio o un allievo dell'astronomo. Nonostante questo margine di incertezza, noi faremo qui riferimento alla breve disputa sul moto terrestre come testo non spurio di Regiomontano non solo perché riteniamo l'attribuzione plausibile fino a prova contraria, ma anche perché siamo persuasi che il suo valore storico-culturale, contestuale ai dibattiti del Cinquecento, non sia inficiato da un'eventuale quanto improbabile errata attribuzione da parte di Schöner.

La biblioteca e le carte del Regiomontano erano preservate in larga parte a Norimberga, sede da lui prescelta negli anni Settanta del Quattrocento per un progetto editoriale volto a restituire testi fondamentali della tradizione scientifica antica e medievale. La lista dei titoli da lui previsti è nota grazie ad un documento a stampa, Haec opera fient in oppido Nuremberga Germania ductu loannis de Monteregio. ${ }^{13}$ II progetto editoriale, interrotto dalla morte prematura dell'iniziatore, prevedeva la pubblicazione di classici quali Euclide, Archimede, Teodosio e Tolomeo oltre ad auctores moderni quali Witelo, Giordano Nemorario e le Theoricae novae planetarum del maestro di Regiomontano, Giorgio Peuerbach (1423-1461). Nella stessa serie sarebbero dovuti apparire anche i suoi lavori matematici. Petreio avrebbe ripreso in parte tale progetto dando alle stampe, tra I'altro, la trigonometria di Regiomontano (De triangulis, 1533) e l'Ottica di Witelo (Perspectiva, 1535).

La disputa che ci interessa si apre con la tesi controversa "Quod moveatur", "che la terra si muova". Regiomontano chiarisce subito che il moto in questione è quello locale e non uno degli altri tipi di moto contemplati dalla filosofia aristotelica, cioè quelli di alterazione, generazione e corruzione. Condizione per poter sostenere che la terra ruoti attorno al proprio asse, mentre i cieli sono in quiete, è che tutti i fenomeni celesti rimangano identici a quelli osservati e spiegati dall'astronomia aristotelico-tolemaica: "Omnia salvari possunt, quae in astris apparent" è presupposto imprescindibile. ${ }^{14}$ Regiomontano riporta che "l'autore della Sfera" rigetta su questa

\footnotetext{
${ }^{12}$ Ernst Zinner, Regiomontanus: His Life and Work, p. 203.

${ }^{13}$ Michela Malpangotto, Regiomontano e il rinnovamento del sapere matematico e astronomico nel Quattrocento (Bari: Cacucci, 2008), pp. 211-217.

${ }^{14}$ È il presupposto metodologico al centro della famosa trattazione di Pierre Duhem, Sōzein ta phainomena: essai sur la notion de théorie physique de Platon a Galilée (Paris: Hermann, 1908). Si veda anche Peter Barker and Bernard R. Goldstein, "Realism and Instrumentalism in Sixteenth Century Astronomy: A Reappraisal", in Perspectives on Science 6/3 (1998), pp. 232-258.
} 
base la dottrina del moto terrestre, con probabile riferimento a Giovanni di Sacrobosco e al suo testo standard per l'insegnamento dell'astronomia sferica medievale. ${ }^{15}$ Egli non pare distinguere qui moto diurno e annuo, nonostante Tolomeo fosse stato attento a separare la confutazione del moto di rotazione da quella relativa al dislocamento della terra dal centro cosmologico. ${ }^{16} \mathrm{Nel}$ primo libro dell'Almagesto egli aveva dovuto fare i conti con la teoria del 'Copernico dell'antichità', di Aristarco di Samo. Regiomontano non si confronta invece con una teoria particolarmente raffinata del moto terrestre. Si limita a riportare che i suoi antichi sostenitori basavano su di un'analogia grossolana tra la terra e la carne allo spiedo l'argomento secondo il quale, come il fuoco non è mosso dal bisogno dell'arrosto, così non è il sole, bensì la terra a doversi muovere spinta dal desiderio del calore dell'astro diurno.

Una volta introdotta la "nota quaestio" Regiomontano sviluppa la sua trattazione attorno a due tesi, o conclusiones, una relativa al moto della terra nella sua interezza e una relativa al moto delle sue parti. ${ }^{17}$ La rotazione da occidente a oriente è rigettata in base ad un noto argomento tolemaico, tratto dal primo libro dell'Almagesto, secondo cui la rapidità del moto dovrebbe produrre un forte vento e lasciarsi indietro tutto ciò che si trovi in aria. Se la terra ruotasse, gli uccelli che desiderassero volare verso oriente incontrerebbero la tenace resistenza dell'aria agitata in direzione contraria. Nubi e volatili dovrebbero scorrere continuamente verso occidente. Inoltre, sostiene Regiomontano sulla base di un classico argomento contro il moto terrestre, il moto verticale risulterebbe impossibile. Se per esempio si gettasse un oggetto in alto, perpendicolarmente al terreno, non potrebbe ricadere nel punto di partenza a causa dello slittamento del suolo.

Il moto della terra quale totalità è dunque bandito. Lo stesso non può dirsi delle parti, le quali, come si afferma nella conclusio secunda, sono in continuo movimento. Regiomontano riprende la dottrina medievale dei 'piccoli moti della terra' descritta da Pierre Duhem nei suoi classici studi su Leonardo da Vinci. ${ }^{18}$ Essa fa riferimento a processi geologici di ridistribuzione della massa terrestre dovuta all'essiccamento della sua superficie e fenomeni di erosione ad opera delle acque. La necessità continua di ristabilire il centro di gravità dell'elemento terrestre fa sì che le parti scorrano senza posa al fine di garantire l'immobilità del tutto. Due corollari fanno seguito a questa tesi. Il primo afferma che il centro della terra non è sempre occupato dalla stessa parte ma è attraversato da parti che si susseguono le une alle altre. Ne consegue, nel secondo corollario, che la materia viene continuamente trasportata dall'alto al basso e viceversa. Regiomontano passa quindi dal moto astronomico della terra, da lui rigettato con decisione, alla discussione, e accettazione, di

\footnotetext{
${ }^{15}$ Si veda il classico Lynn Thorndike, The Sphere of Sacrobosco and Its Commentators (Chicago: The University of Chicago Press, 1949). Si veda anche Matteo Valleriani, "The Tracts of the Sphere: Knowledge Restructured over a Network", in id. (curatore), The Structures of Practical Knowledge (Cham: Springer, 2017), pp. 421-474.

${ }^{16}$ Cf. Pietro Daniel Omodeo e Irina Tupikova, "Cosmology and Epistemology: A Comparison between Aristotle's and Ptolemy's Approaches to Geocentrism," in Spatial Thinking and External Representation: Towards a Historical Epistemology of Space, a cura di Matthias Schemmel (Berlin: Edition Open Access, 2016), pp. 131-158.

${ }^{17}$ La quaestio è nota soprattutto grazie alle dispute di autori medievali come Buridano e Oresme, ampiamente discussi da Marshall Clagett, The Science of Mechanics in the Middle Ages (Madison, Wisconsin: The University of Wisconsin Press, 1959). Ma si veda anche Pierre Duhem, Un précurseur français de Copernic: Nicole Oresme (1377) (Paris: Librairie Armand Colin, 1909).

18 Pierre Duhem, Études sur Léonard de Vinci: ceux qu'il a lus et ceux qui l'ont lu (Paris: Édition des archives contemporaines, 1984), vol. 2, pp. 332-336, "Leonard de Vinci et les origines de la géologie".
} 
una sorta di 'moto convettivo' interno all'elemento terrestre. Prova ne siano le montagne e le rocce, erose dalla pioggia e dalle intemperie o riarse e compattate dai raggi del sole. Le radici degli alberi che restano scoperte sarebbero ulteriore attestazione dello sgretolarsi dei terreni.

La disputa si chiude, come prevede il genere, con un sommario delle conclusioni raggiunte, "sic patet...". Regiomontano ricapitola secondo quale accezione (qualiter) la terra si debba dire immobile e secondo quale possa essere detta invece in movimento. Dal punto di vista astronomico essa è ferma. Non potrà quindi ruotare sui poli al posto della sfera celeste (sicut Spahera). Dal punto di vista della teoria degli elementi si dovrà invece asserire che la terra, o meglio le sue parti, siano mosse dalla loro gravità così come gli altri elementi (acqua, aria e fuoco) il cui moto è più facilmente riscontrabile. Questi avrebbero più propensione ad essere agitati a causa della loro maggiore leggerezza.

Si nega infine, "a ragion veduta" (ad rationem), che il moto terrestre possa "salvare tutti i fenomeni" (quod omnia possint salvari). Regiomontano osserva che le congiunzioni e opposizioni dei pianeti, nonché i loro specifici moti, ne risulterebbero sovvertiti. L'obiezione è bizzarra perché la rotazione della terra al centro del cosmo non avrebbe alcuna conseguenza sulle cosiddette 'teorie dei pianeti'. Solo la rotazione attorno al sole poteva sollevare dubbi circa la possibilità di preservare la teoria planetaria sulla cui base possono essere calcolate e previste congiunzioni, opposizioni e moti dei pianeti. Tolomeo aveva considerato il sovvertimento di tali aspetti una conseguenza dell'eccentricità della terra, ossia della possibilità di un suo spostamento dal centro del cosmo. Sorge quindi la domanda: che la conclusione eccentrica della disputa di Regiomontano segnali un'incipiente riflessione sulla possibilità (ovvero impossibilità) di attribuire alla terra anche il moto annuo?

Regiomontano refuta il moto locale della terra anche nell'Epytoma in Almagestum Ptolemaei (postuma, 1496), che rappresenta il maggior contributo di Regiomontano all'astronomia matematica latina. La conclusio quinta del primo libro è intitolata "Quod terra localem motum non habeat declarare". Essa si limita a considerazioni relative alla rotazione assiale:

Ex superioribus constat terrae non accidere motum rectum. Sic enim medium mundi relinquere cogeretur, quod ante hac prohibuimus. Oporteret denique terram velocissime moveri mole sua id agente, unde reliqua corpora minus gravia terre adiacentia in aere relinquerentur si omnia gravia ad unum niterentur terminum, quod nusquam apparet. Terra demum circularem non habet motum. Si enim circa axem mundi moveretur ab occidente ad orientem, omnia que in aere moverentur semper versus occidentem moveri viderentur. Non enim possent consequi motum terrae. Cuius contrarium in nubibus motis atque avibus sepenumero experimur. Idem quoque accideret: si aerem una cum terra hoc pacto moveri putaveris. Terra postremo circa alium quempiam axem non movetur. Sic enim altitudo poli nobis in terra quiescentibus varia haberetur. Quod cum nemini appareat, terram hac lege moveri non posse constat. ${ }^{19}$

La discussione del solo moto terrestre di rotazione assiale si colloca evidentemente in un clima filosofico e scientifico precopernicano. Vi è comunque un capitolo iniziale dell'Epytoma, la conclusio tertia, dedicato alla dimostrazione della centralità della terra, "Terram in medio mundi sitam esse."

\footnotetext{
${ }^{19}$ Giovanni Regiomontano e Giorgio Peuerbach, Epytoma in Almagestum Ptolomei (Venetiis: Johannes Hamman, 1496), f. a6v.
} 
Esso esclude sin dall'inizio la possibilità che la terra possa mai occupare una posizione eccentrica rispetto al centro cosmologico e quindi, a fortiori, che possa ruotare attorno ad un centro differente da quello occupato dalla terra stessa. ${ }^{20}$

La coda della disputa sul moto terrestre inserisce la discussione all'interno di una cornice astronomica più ampia. Dopo aver trattato della sfericità e dell'immobilità della terra, si legge, occorre passare all'asse del mondo, ai vari circoli della sfera celeste e alla loro proiezione sul globo terrestre. Che la disputa fosse dunque il tassello di un mosaico più vasto? Di un'introduzione generale all'astronomia sferica? Può darsi, ma è più probabile che la conclusione sia un'intromissione di Schöner volta a incastonare il prezioso testo del 'maestro' nel suo Opusculum geographicum. II capitolo primo, precedente l'An terra moveatur, tratta infatti della forma sferica del globo terrestre mentre le tematiche anticipate nella conclusione della disputa, asse terrestre e circoli celesti, vengono affrontate nei capitoli seguenti, tre e quattro.

Anche se la disputa di Regiomontano impallidisce alla luce degli sviluppi teorici del Cinquecento e la profondità dell'argomentazione non pare sufficiente per far fronte all'armamentario che Copernico avrebbe approntato, nondimeno essa costituisce un non trascurabile documento della cultura scientifica di quegli anni. Occorrerà ricordare che la funzione della disputa medievale non era tanto quella di riaffermare verità incontrovertibili quanto quella di soppesarle e sottoporle ad un rinnovato vaglio. Come ha sottolineato la storica dell'università parigina Olga Weijers, "la risposta finale data da un maestro di filosofia [...] alle questioni disputate non era necessariamente vista quale la soluzione definitiva del problema. Costoro [maestri e dottori] fanno spesso mostra di una certa modestia e sono pronti a rivedere la loro opinione." Inoltre "gli argomenti addotti a favore della posizione contraria, quella da refutare, venivano rigettati, certo, ma non venivano disprezzati o considerati privi di valore. Al contrario, essi contribuivano alla discussione, rivelando i vari aspetti del problema e aiutavano a mostrare la non validità della risposta contraria." ${ }^{21}$ Da questo punto di vista la disputa di Regiomontano contro il moto terrestre e la considerazione da parte dei suoi lettori rinascimentali non vanno interpretate nei termini di una chiusura dogmatica. Attestano piuttosto che il moto terrestre era tema 'disputabile' e fu anche di fatto 'disputato' nel tardo Quattrocento e nel primo Cinquecento, all'alba dell'astro nascente di Copernico.

Traduzione

Disputa di Giovanni Regiomontano se la terra si muova o stia ferma. Capitolo II

Si può sostenere che la terra si muove perché tutti i fenomeni legati agli astri possono essere salvati attraverso il moto circolare della terra da Occidente a Oriente. Di conseguenza, se diciamo che la terra viene mossa e il cielo sta fermo, non vi è nulla di incongruo. Al contrario pensa l'autore della

\footnotetext{
${ }^{20}$ Ivi, ff. a5v-a6r.

${ }^{21}$ Olga Weijers, A Scholar's Paradise: Teaching and Debating in Medieval Paris (Turnhout: Brepols, 2015), p. 122 (traduzione propria).
} 
Sphaera. ${ }^{22}$ Una nota questione [nota quaestio] verte sul moto locale e non sul moto di alterazione (ossia di generazione o di corruzione), precisamente se la terra abbia un moto locale [localiter moveatur]. Già alcuni tra gli antichi ritenevano che il cielo stesse fermo e la terra si muovesse in circolo sui suoi poli, compiendo in un giorno una rotazione [revolutio] da Occidente verso Oriente. Così ci si immaginava che la terra fosse come la carne arrostita nello spiedo e il sole come il fuoco che arrostisce. Sostenevano, infatti, che, come il fuoco può fare a meno dell'arrosto, al contrario, non è il sole ad aver bisogno della terra, ma piuttosto la terra del sole.

Prima tesi. La terra non ha un moto circolare da Occidente verso Oriente circa i suoi poli né un moto giornaliero sul proprio centro, come pensavano costoro. ${ }^{23}$ Ciò è abbastanza evidente, perché sarebbe più difficile andare verso Occidente che verso Oriente, cosa che è contro l'esperienza. Ci si aspetterebbe che anche l'aria vicino alla terra si muovesse in modo tale da essere di ostacolo a chi cammina. Persino gli uccelli non potrebbero volare bene verso Oriente [contra orientem] a causa dell'aria che li incalzerebbe e solleverebbe le loro penne. Infatti [non] vediamo gli uccelli volare meglio contro vento [contra ventum] che seguendo il vento [cum vento]. ${ }^{24}$ Allo stesso modo ciò che viene gettato verso l'alto non ritornerebbe al punto di partenza e, per la stessa ragione, vedremmo le costruzioni rompersi a causa di un impeto molto violento. Tuttavia è ancora più evidente che la terra non si muove [non moveatur] di moto giornaliero per il fatto che gli uccelli si vedono muoversi in aria verso Oriente [versus orientem], e similmente fanno le nubi, cosa che non accadrebbe mai se la terra si muovesse in modo tale da dover essere mossa più velocemente tanto da superare essa stessa con il suo movimento il movimento di tutti gli esseri dell'aria, e quindi si vedrebbero gli uccelli e tutte le nubi muoversi verso Occidente [versus occidentem].

Seconda tesi. È evidente che qualsiasi parte della terra si muove di continuo localmente. Infatti, come di continuo la parte arida della terra a causa dei raggi solari si scalda, si consuma, si leviga, così molte piccole parti della terra e anche dell'acqua vengono portate dalla parte arida attraverso i fiumi verso il mare aperto. Da ciò deriva che la parte della terra coperta dall'acqua, che arriva anche a condensarsi e solidificarsi per la freddezza dell'acqua, è più pesante. Perciò conviene che essa si diriga in su verso l'altra tanto a lungo finché il centro di tutto il peso [gravitas] coincida col centro del mondo [medium mundi]. Di conseguenza qualsiasi parte della terra è mossa da un continuo moto locale.

Corollario. Non sempre la stessa parte della terra rimane nel centro del mondo, ma un'altra e così via. ${ }^{25}$

Corollario. Si constata che, dopo una lunga successione di tempo, presupposta la perpetuità del mondo, la parte della terra che per un certo tempo fu al centro del mondo viene in superficie e viceversa. Da ciò si ha la distruzione dei grandi monti e delle rocce, perché le parti della terra meno

\footnotetext{
${ }^{22}$ Probabile riferimento all'opera dell'astronomo parigino del secolo XIII Giovanni Sacrobosco, oppure all'astronomo islamico del secolo IX Al-Farghani, noto in Occidente come Alfraganus.

${ }^{23}$ Costoro, isti nell'originale, si riferisce ai sostenitori della tesi aristotelico-tolemaica dell'immobilità terrestre.

24 Il testo stampato sarebbe: “Nam [non] melius volare videmus aves contra ventum quam cum vento." Riteniamo vi sia un refuso e che, per dare senso alla frase, serva introdurre la particella negativa.

${ }^{25}$ La ristampa anastatica offerta a pp. 37-39 dell'Opera collectanea di Regiomontano a cura di Felix Schmeidler (1972), riporta un testo latino corrotto da una macchia di inchiostro: [a]lia et successive. Abbiamo conferma della nostra integrazione testuale grazie all'ispezione di una copia dell'Opusculum Geographicum in una copia del 1533, probabilmente l'esemplare di Schöner medesimo, custodita alla Dibner Library for the History of Science and Technology, Washington DC.
} 
tenaci vengo erose dalle piogge, mentre rimangono le parti della terra più tenaci che cuociono continuamente per i raggi del sole e ricevono la durezza maggiore. In questo stesso modo, se qualcuno non vuole credere all'erosione della terra, guardi le radici degli alberi antichi nei boschi, e le vedrà già che escono fuori dalla terra, mentre invece un tempo dovevano risiedere dentro la terra. Così è chiaro in quale accezione intendiamo che la terra è immobile, cioè che non si muove in circolo rispetto al proprio centro, in quanto centro della sfera celeste. ${ }^{26}$ In più essa non è in continua alterazione locale a causa del proprio peso, come invece gli altri elementi, i quali sono più leggeri e sono soggetti facilmente all'agitazione e al moto. Si deve ragionevolmente negare che tutte le apparenze ${ }^{27}$ si possono salvare. ${ }^{28}$ Infatti attraverso di esso (moto terrestre) non possono essere conservate né le congiunzioni né le opposizioni dei pianeti e le differenze dei loro moti. Inoltre non possiamo salvare il fatto che vediamo gli uccelli e le nubi di tanto in tanto muoversi verso Oriente [versus orientem], mentre si dovrebbero muovere sempre verso Occidente [versus occidentem].

Abbiamo così ammesso la rotondità e l'immobilità della terra, che insiste sul centro del mondo, cioè il centro di ciascun elemento e delle sfere celesti, senza ulteriori distinzioni di circoli. Ora è tempo di parlare dei circoli della sfera celeste, che si raffigurano tanto nel globo della terra quanto nel cielo, a partire dalla definizione dell'asse del mondo.

Index Nominum

Albategno (Al-Battānī)

Alfragano (Al-Farghān̄i)

Aristotele

Buridano (Buridan, John)

Copernico, Niccolò

Giovanni da Siviglia

Nemorario, Giordano (Jordanus Nemorarius)

Oresme, Nicola

Petreio, Giovanni (Petreius, Johannes)

\footnotetext{
${ }^{26}$ Sphaera significa l'insieme delle sfere celesti.

${ }^{27}$ Moti di corpi celesti e fenomeni legati ad essi visibili ad occhio nudo.

${ }^{28}$ Giustificare con leggi che abbiano portata generali, siano cioè valide per tutti i corpi celesti considerati in un sistema cosmologico di riferimento.
} 
Peuerbach, Giorgio (Georg P.)

Platone da Tivoli

Regiomontano, Giovanni (Johannes Müller)

Retico, Giorgio Gioacchino (Rheticus, Joachim)

Sacrobosco, Giovanni (John Hollywood)

Schöner, Giovanni (Johannes S.)

Tolomeo, Claudio (Claudius Ptolemaeus)

Witelo (Erazmus Ciolek W.) 